\title{
Evidence for preserved direct pupillary light response in Leber's hereditary optic neuropathy
}

\author{
Masato Wakakura, Junko Yokoe
}

\begin{abstract}
Aims/Background-Pupillary light response is usually defective in all types of optic neuropathy. However, the authors have observed in patients with Leber's hereditary optic neuropathy (LHON) relatively normal light response, with consequent misdiagnosis psychogenic visual loss in some cases. To confirm this clinical impression, afferent pupillary defect was assessed by measurement of adjusted constriction amplitude (CA) and escape rate (ER) by infrared videopupillography (Iriscorder-C 2515).
\end{abstract}

Methods-Thirteen consecutive patients (26 eyes) with LHON (average age $27 \cdot 2$ years) were examined; 12 had the mitochondrial DNA 11778 mutation and one the 14484 mutation. Seven of these patients had a positive family history. For comparison, the above rates were determined in 19 patients ( 23 eyes) with idiopathic optic neuritis ( $O N$; average age $35 \cdot 1$ years), 18 patients (19 eyes) with anterior ischaemic optic neuropathy (AION; average age 58.1 years), and 25 volunteers ( 50 eyes) with healthy eyes (average age 39.6 years).

Results-The distribution of visual acuity was essentially the same in all optic neuropathy groups. Reduction in CA and increase in ER were significant in patients with ON and AION, but not in those with LHON. Only slight afferent pupillary defect was evident even 2 years after the onset of LHON. CA in AION and ER in ON were correlated statistically with visual acuity and Humphrey mean threshold deviation, while CA and ER in LHON were not.

Conclusion-Pupillary light response in patients with LHON obviously differs from that in patients with other types of optic neuropathy. LHON appears to be pathophysiologically characterised by well preserved afferent fibres for pupillary light response (probably from $W$ cells). Besides being of pathogenetic interest, the detection of clinical features should facilitate the diagnosis of LHON particularly when family history provides no indication.

(Br f Ophthalmol 1995; 79: 442-446)

Leber's hereditary optic neuropathy (LHON) is a maternally inherited disease characterised by bilaterally acute or subacute central visual loss in young men. Despite the extraordinary development of mitochondrial (mt) DNA analysis in LHON, ${ }^{1}$ the diagnosis of this disorder remains difficult, particularly soon after its onset. There being many patients with an onset interval for bilateral involvement, normal disc appearance, absence of circumpapillary telangiectasic microangiopathy, unconfirmed family history, and/or late onset may be the reasons for this. Colour vision tests may be helpful in detecting the early involvement of LHON, ${ }^{2}$ asymptomatic carriers, and presymptomatic eye. ${ }^{3}$

In visually evoked cortical potentials, Carroll et $\mathrm{al}^{4}$ found abnormal values in some asymptomatic family members. Nevertheless, some LHON patients have been misdiagnosed initially by the authors or others ${ }^{5}$ as psychological visual loss owing to the fact that pupillary light response was nearly normal. LHON would thus appear to be characterised by highly preserved pupillary light response. In this study, pupillary light response was assessed in patients with LHON and the results were compared with those for patients with other types of optic neuropathy and controls by videopupillography.

\section{Subjects and methods}

A review was made of the results of videopupillography of 13 consecutive subjects (26 eyes) with LHON (average age $27 \cdot 2$ years), 19 subjects (23 eyes) with idiopathic optic neuritis (ON, average age $35 \cdot 1$ years), and 18 subjects (19 eyes) with anterior non-arteritic ischaemic optic neuropathy (AION, average age $58 \cdot 1$ years). Each patient group was formed by lumping together the results from left and right eyes for individual subjects. The data were compared with those of 25 healthy volunteers (50 eyes, average age 39.6 years). LHON was the diagnosis for 12 patients based on the $\mathrm{mt}$ DNA mutation at position 11778 . The $\mathrm{mt}$ DNA mutation was noted at position 14484 in the other patient. Seven patients had a positive family history of this disorder. Examination was performed at various stages of optic neuropathy. For a comparative study of three types of optic neuropathy, initial examination results (7-60 days after subjective visual loss) were used. Direct pupillary light response was recorded with an infrared videopupillometer (Binocular Iriscorder Model C-2515, Hamamatsu Photonics Inc, Hamamatsu, Japan) originally developed by Ishikawa et $a{ }^{6}{ }^{6}$ This device consists of an infrared sensitive silicon vidicon camera, microcomputer system for calculating various variables including change in pupillary area, a monitor scope, and printer. Light emitting diodes served as the light source (peak wavelength, $605 \mathrm{~nm}$ ). Photometric 
A

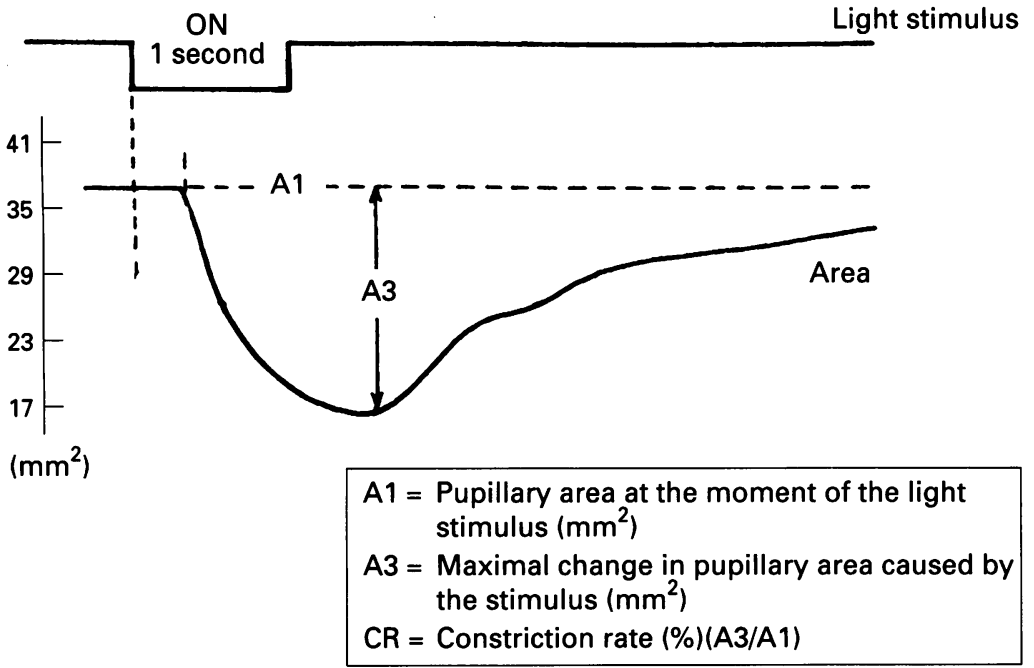

B
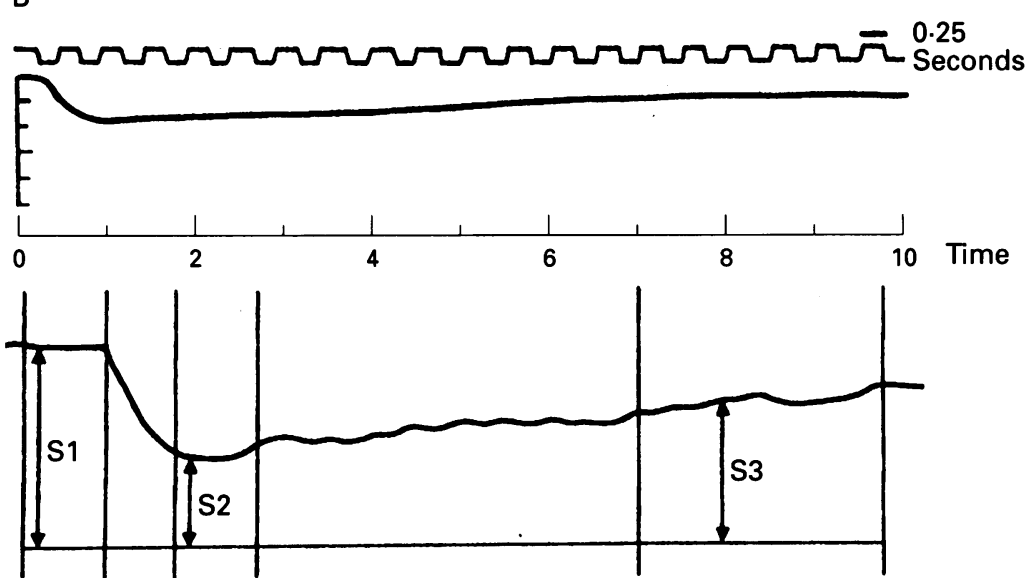

Escape rate (ER) $\quad E R=\mathrm{S} 3-\mathrm{S} 2 / \mathrm{S} 1-\mathrm{S} 2$

Figure 1 Actual recordings of pupillary light response defining adjusted constriction amplitude $(A)$ and escape rate $(B)$.

quantification of the light stimulus was $3.0 \times 10^{-3} \mathrm{~lm} /$ sterad (lumen/steradian) (SD $10 \%$ ).

The light stimulus (diameter on the pupil, $1.2 \mathrm{~mm}$ ) was focused to the centre of the pupil under open loop conditions, the stimulus field being 15 central degrees. All measurements were made following subject adaptation to the dark for at least 15 minutes in a room with only a dim red light source. The subjects were
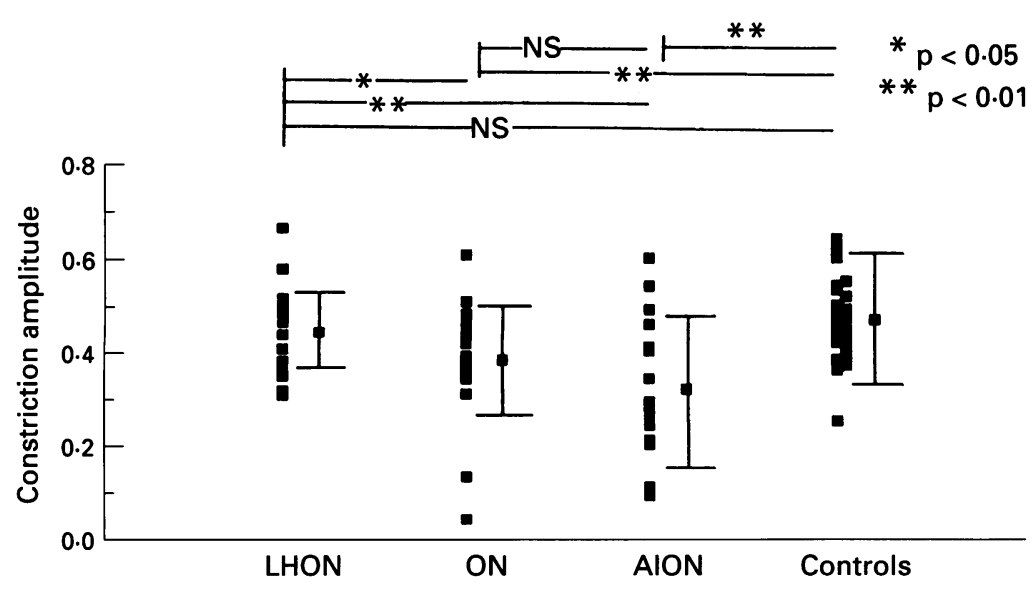

Figure 2 Comparison of constriction amplitude (CA) for the three optic neuropathy groups. LHON=Leber's hereditary optic neuropathy, ON=idiopathic optic neuritis, $A I O N=$ anterior non-arteritic ischaemic optic neuropathy, NS=not significant. instructed not to blink as much as possible and maintain their gaze on a target appearing on a viewing screen inside the pupillometer. Initial and maximal pupillary constrictions were measured from direct pupillary response to light of 1 second duration. Only the direct pupillary response of the affected eye was evaluated. The adjusted constriction amplitude (CA) was expressed as a percentage and the difference in initial and maximal pupillary areas was divided by the initial area. Repetitive pulse stimuli of $250 \mathrm{~ms}$ duration, essentially the same in the case of continuous light stimulus, were used for escape rate (ER) determination. ER, the degree of refractoriness from light stimuli, ${ }^{7}$ was measured and the results are shown in Figure 1. For measurement of corrected visual acuity, Landolt rings were used as the targets, viewing distance was 5 metres, and the results were notated in decimal acuity. Snellen fraction acuity is not used in Japan. The Japanese visual acuity system follows the recommendation of the 11 th International Congress on Ophthalmology (1909). Visual acuity at the start of the examination was ranged from 0.01 to 1.0 . These were comparable with $6 / 600$ and $6 / 6$ in fraction acuity, respectively. Unpaired $t$ tests were conducted for comparison of CA, ER, mean deviation, or foveal sensitivity in each group. Linear regression analysis was carried out for comparison of CA or ER with (1) visual acuity or (2) mean threshold deviation of Humphrey automated perimetry (program 30-2) examined on the same day.

\section{Results}

There were no statistical differences in visual acuity between the three optic neuropathy groups when each group was divided by the acuity at $0 \cdot 2$; the Mann-Whitney test was used. Mean deviation and foveal sensitivity in Humphrey perimetry did not differ statistically among the three patient groups.

\section{CONSTRICTION AMPLITUDE (CA)}

Mean CA in LHON was 0.45 (SD 0.09). In the controls, this value was $0.48(0 \cdot 13)$, and essentially the same as the former $(p>0 \cdot 1)$. In the $\mathrm{ON}$ and AION groups, mean CA was significantly less compared with the controls $(p<0.005)$. Statistical significance was only marginal between CA in LHON and ON $(p=0.01)$. The results for CA are shown in Figure 2. Follow up CA results for more than 2 years after initial examination were obtained for eight eyes of four patients but no definite decrease was noted (Fig 3). Linear regression analysis of CA versus visual acuity showed LHON to have the lowest slope $(0 \cdot 023)$, while AION, the highest slope $(0 \cdot 38)$. The scattergram in Figure 4 indicates CA not to be correlated with visual acuity in LHON $(r=0.05$, $\mathrm{p}=0 \cdot 77)$. The linear relation was relatively high in AION $(r=0.72, \mathrm{p}<0.001)$, but poor in ON $(r=0.27, \mathrm{p}=0.09)$. The correlation coefficients of CA versus visual acuity differed statistically for AION and LHON $(p<0 \cdot 01)$, but no 


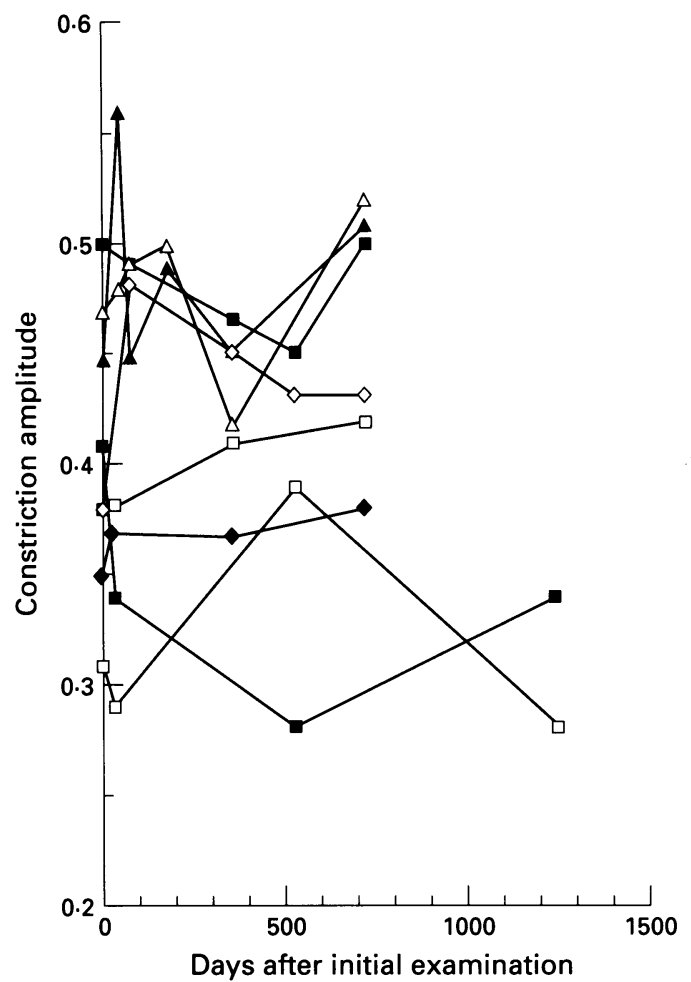

Figure 3 Repetitive examination of constriction amplitude (CA) for more than 2 years after the initial examination in eight eyes with LHON. No definite progressive change in $C A$ could be detected during this period.

other significant difference could be found. The linear relation between $C A$ and Humphrey mean deviation was moderate in AION $(r=0.57, p=0.01)$, but poor in $\mathrm{ON}$ $(r=0.20, \quad \mathrm{p}=0 \cdot 10)$ and LHON $(r=0.05$, $\mathrm{p}=0.82)$ as shown in Figure 5. The correlation coefficients of CA versus mean deviation in the three groups did not differ statistically.

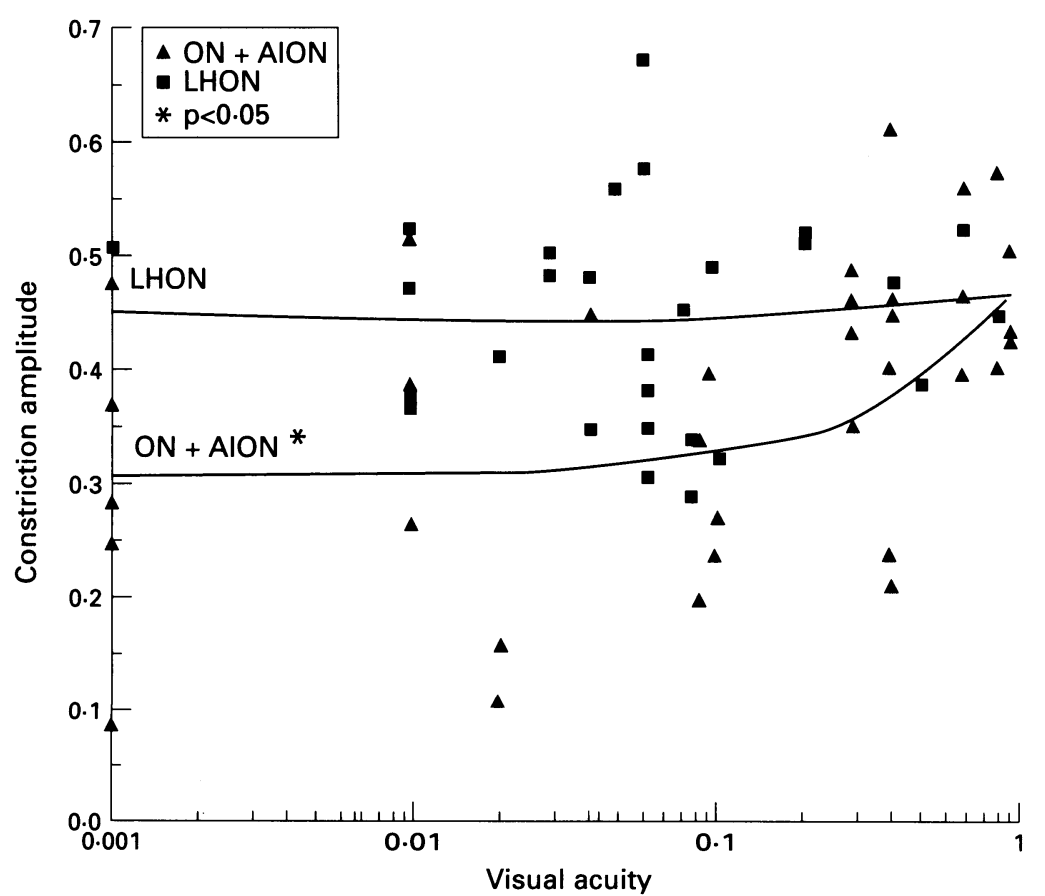

Figure 4 Scattergram showing correlation between visual acuity and constriction amplitude (CA) in LHON and the other types of optic neuropathy (ON+AION). Regression line (LHON): $Y=0.0232 \mathrm{x}+0.441(O N+A I O N): Y=0 \cdot 158 \mathrm{x}+0.308$ Correlation coefficient $(L H O N)=0.05(p=0.77),(O N+A I O N)=0.48(p=0.02)$. For abbreviations see Figure 2.
ESCAPE RATE (ER)

Mean ER in LHON was $0 \cdot 18$ (SD $0 \cdot 18$ ). In the controls, it was $0 \cdot 15(0 \cdot 12)$. Mean ER in LHON was somewhat higher than in the controls, but not statistically so $(p>0 \cdot 1)$. ER in ON and AION was high compared with the controls $(p<0.001)$ and LHON $(p<0.001)$. The ER results for the four groups are shown in Figure 6. ER in eight eyes followed up for more than 2 years showed no significant increase (Fig 7). Linear regression analysis of ER versus visual acuity failed to indicate good correlation in LHON $(r=0 \cdot 25, \mathrm{p}=0 \cdot 12)$ and AION $(0.42, \mathrm{p}=0.09)$, but moderate correlation was shown in ON $(r=0.64, \mathrm{p}=0.001)$. ER in LHON was relatively independent of visual acuity, as shown by the scattergram (Fig 8). The linear relation between ER and the Humphrey mean deviation was moderate in ON $(r=0.52, p=0.009)$, but poor in AION $(r=0.27, \quad \mathrm{p}=0.16)$ and LHON $(r=0.29$, $\mathrm{p}=0 \cdot 12)$. The slope in LHON was lowest among the three optic neuropathy groups (Fig 9 ). The correlation coefficients of these groups did not differ statistically in plots of ER versus visual acuity and mean deviation.

\section{Discussion}

Videopupillographic analysis clearly indicated direct pupillary light response in LHON to be essentially the same as that in normal controls even though the patients exhibited considerable visual loss, in support of our initial clinical impression. Thompson et $a l^{8}$ showed that pupillary deficit was not always related to visual acuity, whereas pupillary defect was significantly related to visual field loss. The static automated perimetry demonstrated relatively good correlation between mean threshold deviation in the central $30^{\circ}$ of vision and loss of pupillary function has been reported in various types of optic neuropathy ${ }^{9}$ and glaucoma. ${ }^{10}$ In these studies, however, an attempt was made to detect relative afferent pupillary defect. In several patients with an onset interval, slight or moderate relative afferent pupillary defect was noted at the early stage of the disease. In this study, no attention was directed to differences in pupillary response in the eyes but rather to direct pupillary response, since LHON is generally symmetrically involved and relative afferent pupillary defect was hardly detected. It would thus appear possible, based on CA and ER results, to detect optic nerve dysfunction in an eye. ${ }^{7}$ Statistical analysis of CA in AION and $\mathrm{ER}$ in $\mathrm{ON}$ clearly indicated these variables to be closely correlated with visual function.

Various clinical characteristics in LHON have been reported in some cases, ${ }^{1} 1112$ including vascular tortuosity, telangiectasic microangiopathy, ${ }^{13}$ and distension of the optic nerve sheath. ${ }^{14}$ Clinical testings looking at visual function and specific for diagnosis of LHON were reviewed by Berninger et al. ${ }^{3}$ Nikoskelainen $e t a l^{2}$ found the Farnsworth-Munsell 100 hue test to be the most sensitive indicator of the early involvement of LHON. Colour vision defects have been observed in some presumed carriers, but not in all. ${ }^{3}$ There is 


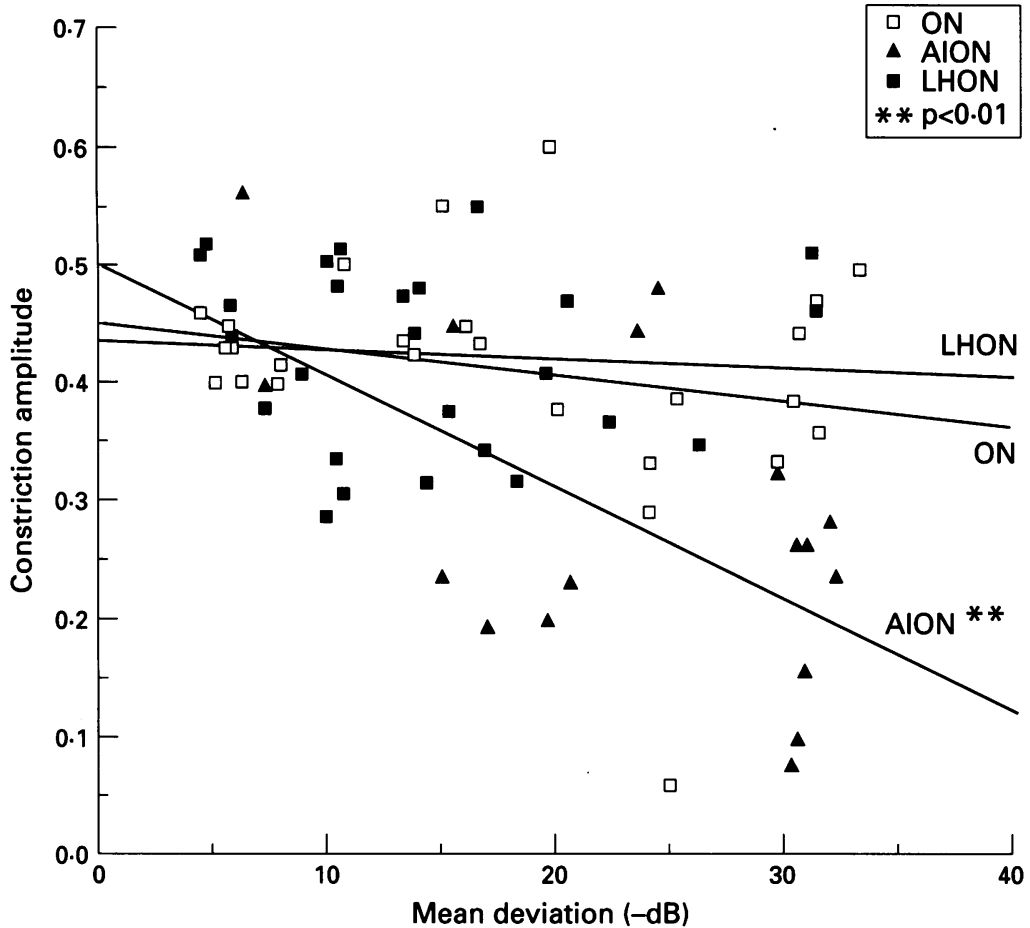

Figure 5 Scattergram showing correlation between Humphrey mean threshold deviation of central 30 degree of vision and the constriction amplitude (CA) in LHON, ON, and AION. Regression line (LHON): $Y=-0.0005 x+0.44,(O N): Y=-0.0021 x+0.45$, (AION): $Y=-0.0094 x+0.51$. Correlation coefficient $(L H O N)=0.05(p=0.82)$, $(O N)=0.20(p=0 \cdot 10),($ AION $)=0.57(p=0.009)$
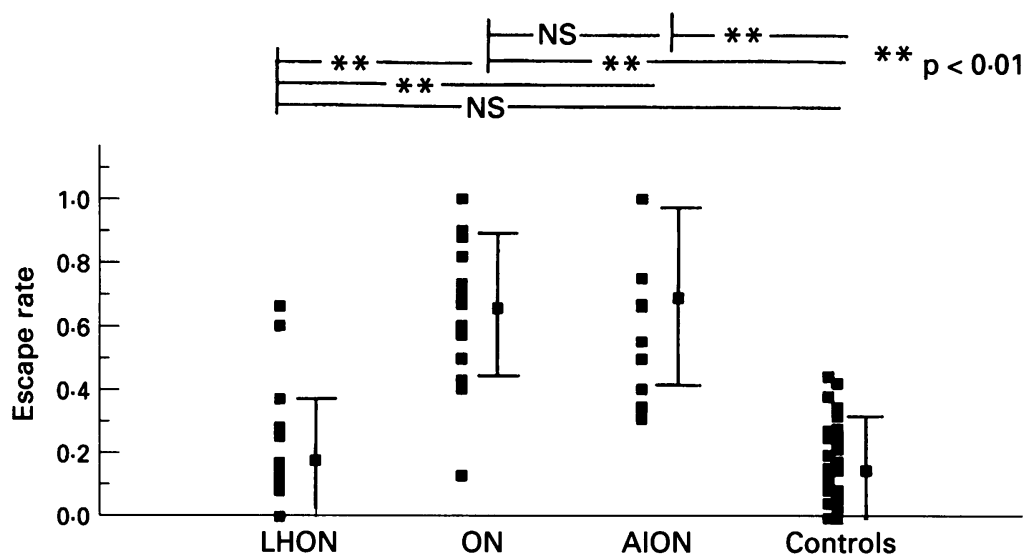

Figure 6 Comparison of escape rate (ER) in the three optic neuropathy groups. For abbreviations see Figure 2.

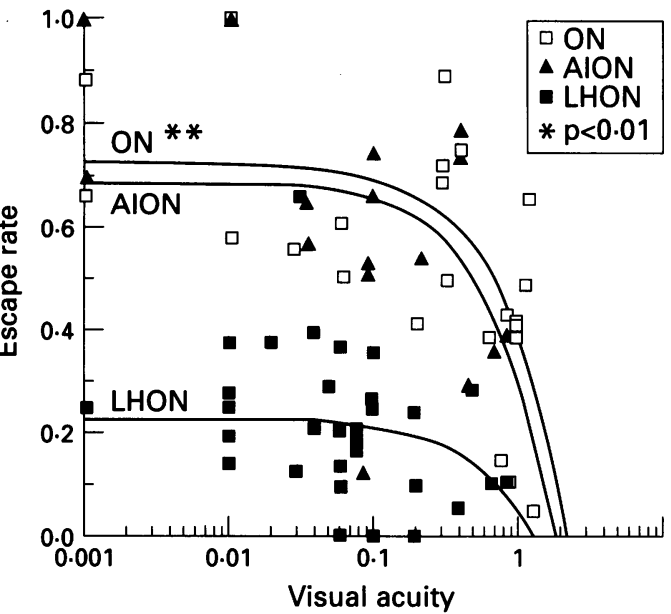

Figure 8 Scattergram showing correlation between visual acuity and escape rate (ER) in LHON and the other types of optic neuropathy. Regression line (LHON):

$Y=-0 \cdot 174 x+0 \cdot 230(O N): Y=-0 \cdot 341 x+0 \cdot 728$,

(AION): $Y=-0.392 \mathrm{x}+0.698$, Correlation coefficient $(L H O N)=0.250(p=0.118),(O N)=0.639(p=0.001)$ $(A I O N)=0.421(p=0.087)$. For abbreviations see

Figure 2.

no agreement as to whether visually evoked cortical potential actually facilitates the detection of presumed carriers or presymptomatic eyes. ${ }^{3}$ Thus, the only clinical feature specific and sensitive for LHON may not confirm the diagnosis.

There is not much in the literature on the preservation of pupillary light response. Nikoskelainen found normal light response in $82 \%$ of 106 patients with LHON, even at the final stage (personal communication). Caecocentral visual field defect reflecting selective involvement of the papillomacular bundle is an important clinical feature in LHON. Nevertheless, results of the present statistical analysis indicate CA and ER in LHON not to be related to the severity of visual disturbance as was also noted in a follow up study for more than 2 years.

W cells are the smallest subclass of retinal ganglion cells. Stone and Fukuda ${ }^{15}$ first suggested in cats that a subgroup of $\mathrm{W}$ cells

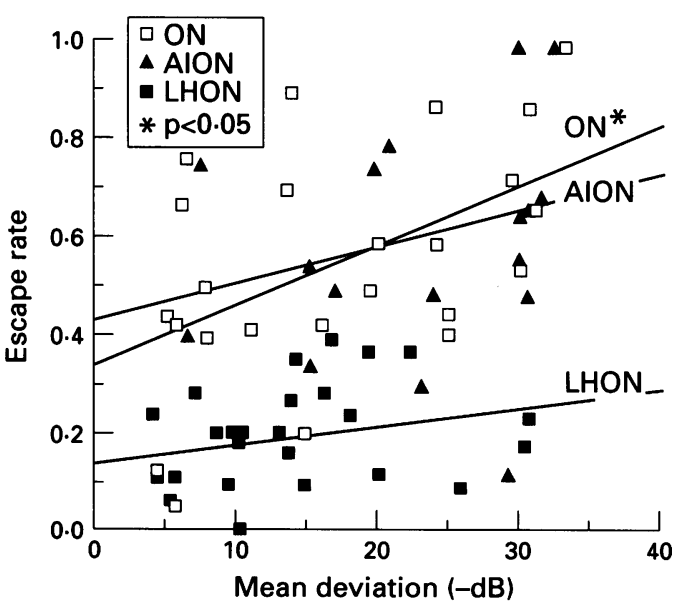

Figure 9 Scattergram showing correlation between Humphrey mean threshold deviation and escape rate $(E R)$ in LHON, ON, and AION. Regression line (LHON): $Y=0.0044 \mathrm{x}+0 \cdot 139,(O N): Y=0.0127 \mathrm{x}+0.340$, (AION): $Y=0.0079 \mathrm{x}+0.433$. Correlation coefficient $(L H O N)=0.289(p=0.122),(O N)=0.516(p=0.009)$ $($ AION $)=0.273(p=0.157)$. For abbreviations see Figure 2.
Figure 7 Repetitive examination of escape rate $(E R)$ for more than 2 years after the initial examination in eight eyes with LHON. No definite progressive change in ER could be detected during this period, except for one eye. 
(tonic on-centre $\mathrm{W}$ cells) functions as a luminance detector and provides input to pupillary light response. This was strongly supported by a precise electrophysiological study. ${ }^{16}$ The pathway of pupillary light response is known to traverse the midbrain. W cells project into the midbrain not only in cats ${ }^{17}$ but also in primates. ${ }^{18}$ Unfortunately, though direct physiological or clinical evidence for this hypothesis still remains unavailable, LHON may be a good example of the possible selective impairment of retinal ganglion cells. W cells would thus appear to remain relatively intact during the course of this disease, in contrast with impaired $\mathrm{X}$ and $\mathrm{Y}$ cells which are essential to visual function. If mitochondrial dysfunction directly causes visual disturbance in LHON, differences in mitochondrial metabolism in these ganglion cells should thus be studied carefully. Kardon et al ${ }^{19}$ noted diseases of the afferent visual system to not necessarily affect the visual threshold and pupillary light reflex in the same way. Rather, mt DNA analysis should be recommended even for cases of optic neuropathy with well preserved pupillary light response.

This work was in part supported by grant-in-aid for scientific research (c), the Japanese Ministry of Education, Science, and Culture (No 04671082) and a grant from Kanagawa Nanbyo Foundation, Kawasaki, Japan. The authors thank Dr Y Mashima and Dr Y Hiida for their DNA analysis.

1 Newman NJ, Wallace DC. The clinical characteristics of pedigrees of Leber's hereditary optic neuropathy with the 11778 mutation. Am $\mathcal{f}$ Ophthalmol 1991; 111: the 11778 .
2 Nikoskelainen E, Sogg RL, Rosenthal AR, Friberg TR, Dorfman LJ. The early phase in Leber hereditary optic norfman LJ. The early phase in Leber hereditary

3 Berninger TA, Bird AC, Arden GB. Leber's hereditary optic atrophy. Ophthalmic Pediatr Genet 1989; 10: 211-27.

4 Carroll WM, Mastaglia FL. Leber's optic neuropathy: a clinical and visual evoked potential study of affected and asymptomatic members of a six generation family. Brain 1979; 102: 559-80.

5 Nakanishi M, Mashima Y, Hiida Y, Suzuki S, Oguchi Y. Two cases of Leber's hereditary optic neuropathy diagnosed as psychogenic visual loss. Ganka (Ophthalmology) (Tokyo) 1994; 36: 811-4.

6 Ishikawa S, Naito $M$, Inaba $K$. A new videopupillography. Ophthalmologica 1970; 160: 248-59.

7 Ishikawa S. A new binocular infrared videopupillography. Shinkeiganka (Neuro-ophthalmol fpn) (Sagamihara) 1986; 3: $235-40$

8 Thompson HS, Cox PMTA, Corbett JJ. The relationship between visual acuity, pupillary defect, and visual field loss. Am $\mathcal{f}$ Ophthalmol 1982; 93: 681-8.

9 Johnson LN, Hill RA, Bartholomew MJ. Correlation of afferent pupillary defect with visual field loss on automated perimetry. Ophthalmology 1988; 95: 1649-55.

10 Brown RH, Zilis JD, Lynch MG, Sanborn GE. The afferent pupillary defect in asymmetric glaucoma. Arch Ophthalmol 1987; 105: $1540-3$

11 Johns DR, Hehler KL, Miller NR, Smith KH. Leber's hereditary optic neuropathy: clinical manifestations of the 14484 mutation. Arch Ophthalmol 1993; 111: 495-8.

12 Wakakura M. Retina and optic nerve disorders. Curr Opin Ophthalmol 1993; 4: 16-21.

13 Nikoskelainen E, Hoyt WF, Nummelin K, Schatz $H$ Fundus findings in Leber's hereditary optic neuroretinopathy. Arch Ophthalmol 1984; 102: 281-9.

14 de Gottrau P, Büchi ER, Daicker B. Distended optic nerve sheaths in Leber's hereditary optic neuropathy. nerve sheaths in Leber's hereditary optic

15 Stone J, Fukuda Y. Properties of cat retinal ganglion cells: a comparison of W-cells with X-and Y-cells. $\mathcal{f}$ Neurophysiol 1974; 37: 722-48.

16 Hult born $\mathrm{H}$, Mori $\mathrm{K}$, Tsukahara $\mathrm{N}$. The neuronal pathway subserving the pupillary light reflex. Brain Res 1978; 159 255-67.

17 Fukuda Y, Stone J. Retinal distribution and central projections of Y-, X-, and W-cells of the cat's retina. f Neurophysiol 1974; 37: 749-72.

18 Perry VH, Cowey A Retinal ganglion cells that project to the superior colliculus and pretectum in the macaque the superior coliculus and pretectum in

19 Kardon RH, Haupert CL, Thompson HS. The relationship between static perimetry and the relative afferent pupillary defect. Am f Ophthalmol 1993; 115: 351-6. 